\title{
Implante por Cateter de Bioprótese Valvular Aórtica para Tratamento de Estenose Valvar Aórtica Grave em Pacientes Inoperáveis sob Perspectiva da Saúde Suplementar - Análise de Custo-Efetividade
}

\author{
Marcelo Cartaxo Queiroga', Alvaro Mitsunori Nishikawa², Luciano Morganti Paladini², \\ Pedro Alves Lemos Neto ${ }^{4}$, Fabio Sândoli Brito Júnior ${ }^{5}$, Rogério Sarmento-Leite ${ }^{6}$, Christiane Bueno \\ Otávio Augusto C. Clark ${ }^{8}$ J. Antonio Marin-Neto ${ }^{9}$, J. Eduardo Sousa ${ }^{10}$
}

\begin{abstract}
RESUMO
Introdução: $\mathrm{O}$ implante por cateter de bioprótese valvular aórtica (TAVI, do inglês transcatheter aortic valve implantation) constitui nova modalidade de tratamento destinada, sobretudo, aos pacientes com elevado risco cirúrgico. Para esses pacientes, o TAVI resultou em aumento da sobrevivência e melhora da qualidade de vida, comparativamente ao tratamento padrão (medicamentoso, com ou sem valvuloplastia aórtica percutânea). Nosso objetivo foi realizar análise de custo-efetividade da implementação do TAVI no Sistema de Saúde Suplementar brasileiro. Métodos: Foram desenvolvidos um modelo preditivo, para avaliar custo-efetividade real do procedimento em longo prazo, e uma regressão de Weibull com tempo horizonte de 5 e 10 anos, para estimar dados de sobrevida por mais de 24 meses. Adicionalmente, foi desenvolvido modelo de Markov sequencial e determinístico. Resultados foram expressos como razão de custo-efetividade incremental (RCEI) por anos de vida ganhos e anos de vida livres de progressão. Resultados: Para o cenário padrão, no qual o custo da prótese valvular foi estipulado em R\$ 65 mil, o valor da RCEI (custo/ano de vida salvo) em 5 anos foi de R\$90.161,29. Alterando-se o tempo horizonte para 10 anos, esse valor diminuiu para $\mathrm{R} \$ 55.130,84$.
\end{abstract}

\begin{abstract}
Transcatheter Aortic Valve Implantation for the Treatment of Severe Aortic Valve Stenosis in Inoperable Patients under the Perspective of the Brazilian Supplemental Health System Cost-Effectiveness Analysis

Background: Transcatheter aortic valve implantation (TAVI) is a new modality of treatment especially dedicated to patients with high surgical risk. In these patients, TAVI increased survival and improved quality of life when compared to standard treatment (drug therapy with or without percutaneous aortic balloon valvuloplasty). Our objective was to perform a cost-efficacy analysis of the implementation of TAVI in the Brazilian Supplemental Health System. Methods: We developed a predictive model to assess the cost-effectiveness of the procedure in the long-term, and a Weibull regression analysis with a time horizon of 5 and 10 years, to estimate survival data for over 24 months. In addition, a deterministic sequential Markov model was developed. Results were expressed as incremental cost-effectiveness ratio (ICER) per years of life saved and progression-free years of life. Results: In a standard scenario,
\end{abstract}

' Doutorando. Diretor Técnico do Serviço de Hemodinâmica e Cardiologia Intervencionista (Cardiocenter) do Hospital Unimed João Pessoa. João Pessoa, PB, Brasil.

${ }^{2}$ Farmacêutico. Farmacoeconomista da Evidências - Credibilidade Científica. Campinas, SP, Brasil.

${ }^{3}$ Médico oncologista. Consultor em Avaliações de Tecnologia em Saúde da Evidências - Credibilidade Científica. Campinas, SP, Brasil. ${ }^{4}$ Livre-docente. Diretor do Serviço de Hemodinâmica e Cardiologia Intervencionista do Instituto do Coração do Hospital das Clínicas da Faculdade de Medicina da Universidade de São Paulo. São Paulo, SP, Brasil.

${ }^{5}$ Doutor. Coordenador médico da Cardiologia Intervencionista do Hospital Israelita Albert Einstein. São Paulo, SP, Brasil.

${ }^{6}$ Doutor. Diretor técnico do Laboratório de Hemodinâmica e Cardiologia Intervencionista do Instituto de Cardiologia do Rio Grande do Sul. Porto Alegre, RS, Brasil.

\footnotetext{
${ }^{7}$ Jornalista. MsC da Evidências - Credibilidade Científica. Campinas, SP, Brasil.

${ }^{8}$ Doutor. Médico oncologista. Diretor da Evidências - Credibilidade Científica. Campinas, SP, Brasil.

${ }^{9}$ Livre-docente. Diretor da Unidade de Hemodiâmica e Cardiologia Intervencionista do Hospital das Clínicas da Faculdade de Medicina de Ribeirão Preto da Universidade de São Paulo. Ribeirão Preto, SP, Brasil. ${ }^{10}$ Livre-docente. Diretor do Centro de Intervenções em Doenças Estruturais do Coração do Instituto Dante Pazzanese de Cardiologia. São Paulo, SP, Brasil.
}

Correspondência: Marcelo A. C. Q. Lopes. Av. Max Zagel, 91 - Camboinha - Cabedelo, PB, Brasil - CEP 58310-000

E-mail: mqueiroga@terra.com.br

Recebido em: 1\%/6/2013 - Aceito em: 6/8/2013 
Conclusões: O modelo apontou que o TAVI apresenta efetividade superior e maior custo incremental. Além disso, a incorporação do TAVI no Rol de Procedimentos e Eventos em Saúde da Agência Nacional de Saúde Suplementar acarretaria impacto orçamentário incremental nos próximos 5 anos, variando de $R \$ 70$ milhões a $R \$ 121$ milhões, compatível com o de outras tecnologias já incorporadas no âmbito da Saúde Suplementar.

DESCRITORES: Estenose da valva aórtica. Implante de prótese de valva cardíaca. Bioprótese. Cateteres. Saúde Suplementar. Análise custo-benefício.

A estenose aórtica (EA) é hoje a valvopatia mais comum entre os idosos, com prevalência estimada de até $5 \%$ nos indivíduos com mais de 75 anos de idade. O relativo envelhecimento atual da população global faz com que essa patologia adquira ainda mais importância social, com impacto significante em políticas de saúde pública. ${ }^{1,2}$

Tipicamente, após longo período de latência clínica, surgem sintomas de angina, síncope e insuficiência cardíaca. A sobrevivência após a manifestação dos sintomas, sem cirurgia de troca valvar, é baixa, sendo de $60 \%$ em 1 ano e de $32 \%$ em 5 anos, ${ }^{3,4}$ com elevado risco de morte súbita. ${ }^{5}$

O tratamento padrão para essa condição é a cirurgia de troca valvar que, em estudos observacionais, mostrou-se capaz de aumentar a sobrevivência e meIhorar os sintomas, comparativamente ao observado em coortes históricas de pacientes tratados sem cirurgia. ${ }^{5-7}$ O procedimento cirúrgico é considerado seguro, com taxa de complicações média em torno de 4 a 5\%, e podendo ser menor que $1 \%$ em pacientes com idade abaixo de 70 anos. $^{8}$

Entretanto, um subgrupo expressivo tem risco cirúrgico elevado, tanto pela idade avançada quanto por associação de comorbidades, tornando-o inelegível para a cirurgia. As taxas de inelegibilidade giram em torno 30 a $40 \%$ dos pacientes. ${ }^{9,10}$ No Brasil, não há dados publicados avaliando essas estatísticas. Para esse grupo de pacientes sem perspectivas de tratamento cirúrgico, o tratamento constitui-se de suporte clínico e medicamentoso, e de valvuloplastia aórtica percutânea com cateter balão. Tais recursos terapêuticos, mesmo associados, têm efetividade limitada e não são capazes de melhorar a sobrevivência dos pacientes., ${ }^{3,6,11} \mathrm{O}$ prognóstico é ominoso, com mortalidade em 1, 2 e 3 anos da ordem de $45 \%, 65 \%$ e $77 \%$, respectivamente. ${ }^{12}$

O implante por cateter de bioprótese valvular aórtica (TAVI, do inglês transcatheter aortic valve implantation) where the cost of the valvular prosthesis was estimated as $\mathrm{R} \$ 65.000,00$, the ICER value (cost/year of life saved) in 5 years was $R \$ 90.161,29$. When the time horizon was adjusted for 10 years, this amount decreased to R\$ 55.130,84. Conclusions: The model indicated that TAVI has superior effectiveness and higher incremental cost. Furthermore, the incorporation of TAVI in the List of Health Procedures and Events of the Brazilian Supplemental Health System would have an incremental budgetary impact over the next 5 years, ranging from $\mathrm{R} \$ 70$ millions to R\$ 121 millions, consistent with other technologies which have already been incorporated by the system.

DESCRIPTORS: Aortic valve stenosis. Heart valve prosthesis implantation. Bioprosthesis. Catheters. Supplemental Health. Cost-benefit analysis. constitui nova técnica introduzida com sucesso para o tratamento dos pacientes considerados inoperáveis. Seu principal objetivo é restaurar a função valvar aórtica por meio de técnicas minimamente invasivas, evitando, assim, a anestesia geral e os procedimentos cirúrgicos, como a esternotomia mediana, o pinçamento aórtico e a circulação extracorpórea. ${ }^{13,14}$ No Brasil, o TAVI foi introduzido em 2008 e, desde então, vem sendo realizado em diferentes hospitais da rede de saúde suplementar nas diversas regiões do país. Na atualidade, já foram realizados aproximadamente 700 procedimentos desse tipo no país.

Diversas entidades internacionais recomendam e aprovam o uso de TAVI para a EA em pacientes inoperáveis, como o National Institute for Health and Clinical Excellence (NICE) britânico, ${ }^{15}$ o Health Quality Ontario $^{12}$ e o National Health Committee, da Nova Zelândia. ${ }^{16}$ No Brasil, tanto a Sociedade Brasileira de Hemodinâmica e Cardiologia Intervencionista $(\mathrm{SBHCI})^{6}$ quanto a Sociedade Brasileira de Cardiologia (SBC) ${ }^{11}$ recomendam o TAVI para o grupo de pacientes considerados inoperáveis. Todavia, essa importante técnica ainda não foi incorporada no rol de políticas de saúde do Brasil.

O presente estudo teve como objetivo realizar análise econômica do impacto da incorporação do TAVI destinado inicialmente aos pacientes portadores de EA no Sistema de Saúde Suplementar Brasileiro, avaliando seu custo-efetividade em pacientes não elegíveis ao tratamento cirúrgico e determinando seu impacto orçamentário.

\section{MÉTODOS}

As evidências atuais associadas à efetividade do TAVI no tratamento de pacientes com EA grave são restritas a um curto período. Foi desenvolvido um modelo preditivo, com a finalidade de avaliar, em longo prazo, o custo-efetividade dessa modalidade terapêutica, objetivando aquilatar seus reais benefícios, bem como 
os custos incrementais decorrentes da implementação desse tratamento. Os dados de efetividade foram extraídos do único estudo clínico prospectivo randomizado publicado: o PARTNER coorte B. ${ }^{17}$

\section{População alvo}

Pacientes acometidos por EA grave sintomática sem perspectiva cirúrgica.

\section{Tempo horizonte}

Foi considerado um tempo horizonte de 60 meses (5 anos) por ser mais adequado à captura de dados de custo e benefício clínico do paciente, uma vez que dados em períodos mais prolongados são escassos e que uma modelagem para extrapolação desses dados poderia causar excessivo viés nos resultados.

\section{Perspectiva}

A análise foi conduzida sob a perspectiva do Sistema de Saúde Suplementar Brasileiro e foram considerados apenas custos diretos. Custos indiretos, como perda de produtividade devido à doença, não foram considerados.

\section{Regressão de Weibull}

Com a finalidade de estimar dados de sobrevivência por mais de 24 meses, foi desenvolvida uma regressão de Weibull com tempo horizonte de 5 e 10 anos. A partir dos resultados do estudo PARTNER, ${ }^{17}$ foram coletados dados de sobrevivência dos pacientes em ambas as modalidades de tratamento, a cada 3 meses. Esses dados foram utilizados para desenvolver a curva de sobrevivência projetada com a regressão de Weibull, obtendo-se estimativas de dados de sobrevivência em até 10 anos (Figura 1).

\section{Modelo de Markov}

Adicionalmente, o presente modelo utiliza os dados de curto prazo disponíveis e se fundamenta neles com a finalidade de prever os custos por meio do aumen-

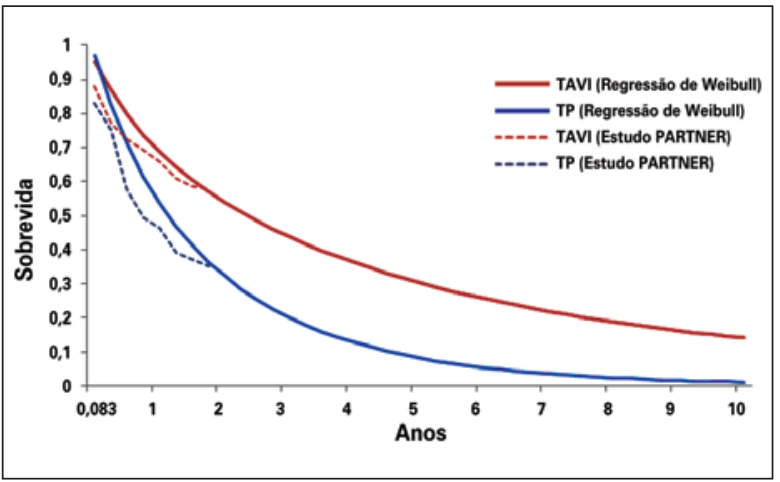

Figura 1. Regressão de Weibull para os dados de sobrevivência projetados para 10 anos nos grupos com tratamento padrão (TP) e com o implante por cateter de bioprótese valvular aórtica (TAVI), a partir de dados do estudo PARTNER. ${ }^{17}$ to do tempo de vida e de benefícios para a saúde associados a diferentes opções de tratamento. Assim, foi desenvolvido um modelo de Markov sequencial e determinístico, com um elemento aleatório opcional. A partir dos dados de sobrevivência dos pacientes obtidos do estudo PARTNER ${ }^{17}$ e da respectiva estimativa de sobrevivência ao longo dos demais anos por meio da regressão de Weibull, a estrutura do modelo envolveu dois estados de saúde: sobrevivência e morte (Figura 2).

Como os dados de sobrevivência obtidos a partir do estudo PARTNER foram baseados em observação de mortes por todas as causas, optou-se por construir um modelo simples (dois estados de saúde). Para tanto, foram considerados todos os custos e probabilidade de comorbidades (acidente vascular cerebral - AVC; complicações graves, como insuficiência renal e implante de marca-passo artificial cardíaco; consultas médicas e hospitalizações). Cada ciclo foi estimado em um tempo horizonte de 3 meses e, para cada ciclo, as probabilidades de mortalidade foram se alterando ao longo do tempo, de acordo com dados do estudo PARTNER e a projeção de Weibull.

Os parâmetros do modelo incluem as durações médias em que o paciente se mantém em cada estado de saúde, bem como os custos associados a tais estados. O modelo foi estruturado em plataforma Microsoft Exce $^{\circledR}$. Ele utiliza ciclos trimestrais com probabilidades para a possibilidade de um paciente mudar de estado de saúde durante cada período. Todas as consequências são relatadas conforme o custo "esperado" e os resultados sobre o estado de saúde de cada paciente.

No modelo, o horizonte de planejamento utilizado foi o tempo de vida do paciente. Por isso, os resultados sobre o estado de saúde e os custos a ocorrerem futuramente têm menos peso que os presentes. Foram aplicados, desse modo, os descontos dos resultados globais sobre o estado de saúde e os custos respectivos. As diretrizes do Ministério da Saúde recomendam que custos e benefícios sejam descontados em 5\%.

\section{Custos}

Foram considerados apenas os custos médicos diretos, como custos de medicamentos, procedimentos e internação hospitalar, coletados sob a perspectiva do Sistema de Saúde Suplementar Brasileiro para o ano de 2013. O consumo de recursos considerado baseou-se em dados de estudo econômico pré-planejado, condu-

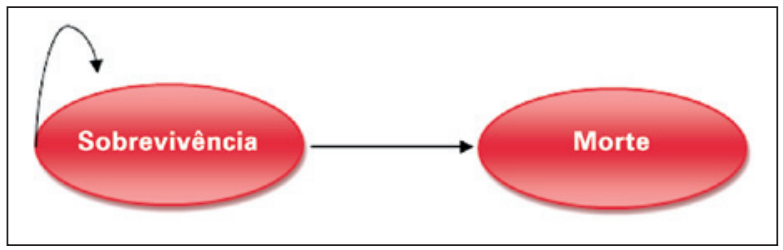

Figura 2. Estrutura do modelo de Markov. 
zido paralelamente ao estudo PARTNER coorte $B,{ }^{18}$ e o padrão de tratamento medicamentoso se fundamentou em registro incluindo pacientes portadores de EA grave e sintomática de alto risco. ${ }^{19}$

Embora no estudo PARTNER 83,8\% dos pacientes do grupo controle tenham se submetido à valvuloplastia aórtica percutânea com cateter balão, esse número foi considerado não generalizável para o cenário de tratamento observado no Brasil, onde, de acordo com o Banco de Dados do Sistema Único de Saúde (DATASUS), apenas 121 procedimentos de valvuloplastia aórtica percutânea com cateter balão foram realizados em 2011. Por meio de um painel de especialistas indicados pela SBHCl, ${ }^{6}$ estimou-se que apenas $30 \%$ da população do grupo controle realizaria esse procedimento (parâmetro variado na análise de sensibilidade).

Como o emprego de valvuloplastia aórtica percutânea com cateter balão não altera a sobrevivência dos pacientes adultos portadores de EA grave sintomática não operável, ${ }^{3,10}$ considerou-se que os dados de efetividade do estudo PARTNER ainda prevalecem, mesmo estimando um menor uso daquele procedimento na população brasileira do presente estudo econômico.

Levando em conta a perspectiva adotada na análise e visando estabelecer os custos farmacêuticos mensais da doença, os valores médios de compra dos medicamentos não foram computados, uma vez que, normalmente, os planos de saúde não reembolsam pacientes pelos medicamentos utilizados no braço de tratamento padrão (amiodarona, furosemida e digoxina), cabendo ao paciente arcar com os devidos custos.

Com base em dados de mercado, estimou-se o custo da prótese utilizada para o TAVI em R\$ 65 mil e do procedimento médico em $\mathrm{R} \$ 23.518,42$, obtido a partir de levantamento de microcosting (técnica de avaliação que envolve a coleta de dados detalhados sobre a entrada de recursos utilizados e o valor desses recursos). Os custos decorrentes de eventos dos tratamentos de pacientes com EA grave desse modelo foram obtidos a partir de painel de especialistas para coleta de uso de recursos, usando fontes referentes ao Sistema de Saúde Suplementar, como a quinta edição da Classificação Brasileira Hierarquizada de Procedimentos Médicos (CBHPM), a Câmara de Regulação do Mercado de Medicamentos (CMED), Simpro e outros (Tabela 1).

\section{Razão de custo-efetividade incremental}

O custo adicional por unidade extra de benefício obtido, conhecido como análise incremental (ou marginal), e seus resultados são apresentados como razões de custo-efetividade incrementais (RCEIs). Os modelos foram usados para estimar os resultados (anos de vida ganhos) e custos para se orientarem os pacientes quanto a cada alternativa de tratamento. Os RCEls foram calculados da seguinte forma:

\section{$R C E I=\frac{\text { Custo tratamento }- \text { Custo alternativo }}{\text { Efeito tratamento }- \text { Efeito alternativo }}$}

Sendo: RCEI: razão de custo-efetividade incremental; custo: custos (em reais); efeito: efetividade (em anos de vida ganhos).

\section{Análise de sensibilidade}

A análise de sensibilidade univariada foi realizada nas duas principais variáveis do modelo: no tempo horizonte dessa análise de custo-efetividade e no custo de incorporação da prótese.

Em razão de as distribuições terem sido ajustadas aos parâmetros fundamentais no modelo, cada um dentre os 5 mil pacientes replicados na análise apresentou uma trajetória diferente da doença e, assim, resultados e custos variados. Dessa maneira, também foi conduzida uma análise probabilística adicional para avaliar a variabilidade dos resultados e a conclusão sobre a possibilidade de que uma determinada intervenção apresentasse custo-efetividade. Todas as distribuições no modelo foram testadas de forma independente.

Todos os custos de tratamento (medicamentoso, procedimento com cateter balão e TAVI) foram variados em $\pm 20 \%$ utilizando-se variação gama, assim como custo de hospitalização e consultas médicas. As taxas de implante de marca-passo, AVC, complicações graves, bem como número de hospitalizações e consultas médicas, foram variadas em $\pm 25 \%$. Dados de efetividade dos tratamentos analisados foram variados em $\pm 10 \%$. Seguindo as diretrizes do Ministério da Saúde, a taxa de desconto foi variada entre zero e $10 \%$.

\section{RESULTADOS}

Ao se empregar o TAVI no tratamento de pacientes inoperáveis com EA grave, o custo total estimado do tratamento, em 5 anos, foi de R\$123.019,76. Esse valor incluiu: custos do procedimento, prótese valvular, consultas com especialistas, hospitalização e gastos com eventos adversos. Já o custo para o tratamento padrão (medicamentoso) foi estimado em R\$35.815,12,

TABELA 1

Custo de eventos

\begin{tabular}{lc}
\hline Procedimento & Custo (R\$) \\
\hline Insuficiência renal (com diálise) & $68.493,35$ \\
Implante de marca-passo & $13.203,26$ \\
Acidente vascular cerebral & $4.722,08$ \\
Complicações vasculares graves & $6.001,15$ \\
Hospitalizações (por outras causas) & $1.503,25$ \\
Consultas médicas & 63,00 \\
\hline
\end{tabular}


no qual se agregaram os custos com especialistas e hospitalizações, entre outros. Os valores foram obtidos por meio de um estudo de microcosting para avaliar o custo total do procedimento, considerando as tabelas atualmente em prática pelas operadoras de planos de saúde.

Os pacientes tratados com TAVI apresentam maior expectativa de vida em comparação aos pacientes que realizam apenas a terapêutica conservadora. Em 5 anos de análise, no grupo que recebeu o implante da prótese valvular por cateter, a média de sobrevivência foi de 2,5 anos de vida. No grupo que recebeu somente o tratamento padrão, a sobrevivência média foi de 1,53 anos de vida.

Para o cenário padrão, no qual o custo do TAVI foi estipulado em R\$ 65 mil, o valor do RCEI (custo/ ano de vida salvo) em 5 anos foi de $R \$ 90.161,29$. No entanto, alterando-se o tempo horizonte para 10 anos, o valor do RCEI diminuiu para R\$ 55.130,84.

Adicionalmente, alterou-se o valor da prótese valvular entre R\$ $30 \mathrm{mil}$ e R\$ $65 \mathrm{mil}$ (valor do caso base). Os resultados de RCEI dessa análise no tempo horizonte de 5 anos, com essa alteração do custo da prótese valvular, variaram entre $\mathrm{R} \$ 53.975,00$ e R\$ 90.161,29. Os valores para cada alteração estão descritos na Figura 3.

Também foi conduzida uma análise probabilística adicional, cujos resultados foram avaliados e classificados em: quadrante 1 (efetividade incremental $>0$ e custo incremental $>0$ ); quadrante 2 (efetividade incremental $<0$ e custo incremental $>0$ ); quadrante 3 (efetividade incremental $<0$ e custo incremental $<0$ ) e quadrante 4 (efetividade incremental $>0$ e custo incremental $<0$ ).

Verificou-se que a maior parte das simulações possui resultados no quadrante 1 (99,9\%), ou seja, na simulação realizada, a quase totalidade dos pacientes utilizando o TAVI, comparativamente ao tratamento padrão, caracteriza-se por maior efetividade e também por custo incremental, evidenciando robustez no modelo da análise.

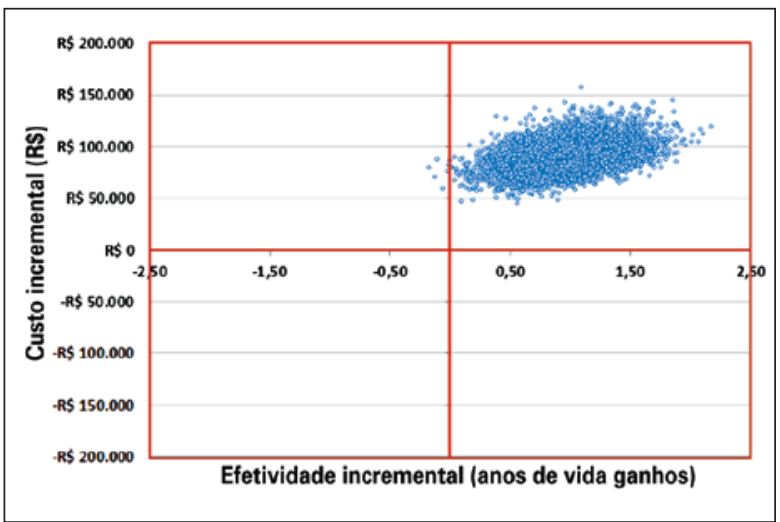

Figura 3. Plano probabilístico de custo-efetividade.
Em adição, foi desenvolvida uma análise de impacto orçamentário (BIM, do inglês Budget Impact Models) com o intuito de simular o impacto financeiro da introdução do TAVI no Sistema de Saúde Suplementar. Essa análise foi feita comparando-se o custo dos primeiros anos de tratamento de pacientes inoperáveis com EA grave. Assim, o impacto no orçamento dessa nova tecnologia compara o custo de aquisição da bioprótese valvular (para esse grupo de pacientes) a outra opção terapêutica vigente.

O modelo foi criado em plataforma Microsoft Excel ${ }^{\circledR}$, seguindo as diretrizes de análises de impacto orçamentário publicadas em 2007 pela International Society for Pharmacoeconomics and Outcomes Research (ISPOR). Tal modelo estima o impacto orçamentário, ao combinar dados epidemiológicos, hipóteses de evolução do uso das várias terapias disponíveis (fluxo de tratamento), recursos utilizados e os custos do tratamento medicamentoso e de eventos adversos associados, comparando dois ou mais cenários distintos.

Para os cenários hipotéticos divisados (cenário base e cenário alternativo), o perfil de mercado para a nova técnica (TAVI), ao longo de 5 anos, foi estimado a partir da tendência projetada de uso, uma vez que não existem dados de "mundo real" para os cenários em questão. No cenário base, partiu-se da premissa de que o implante por cateter da prótese valvular deve ter um perfil de emprego obtido por meio da opinião do painel de especialistas já nominado. Como análise de sensibilidade, foi considerado um cenário extra, nomeado "cenário alternativo", no qual foi estimado um perfil de emprego do TAVI maior após a incorporação da nova tecnologia (Figura 4).

Para cada ano do horizonte de 5 anos do modelo, assumiu-se, no cenário atual, que a totalidade de pacientes elegíveis deve ser inteiramente tratada com o padrão terapêutico atual (medicamentos e dilatação por cateter balão).

A análise do impacto orçamentário mostrou que, no cenário atual, a estimativa dos gastos totais, ao

\begin{tabular}{|l|c|c|c|c|c|}
\hline & \multicolumn{5}{|c|}{ Cenário base } \\
\cline { 2 - 6 } & Ano 1 & Ano 2 & Ano 3 & Ano 4 & Ano 5 \\
\hline Padrão terapêutico atual & $70 \%$ & $65 \%$ & $60 \%$ & $55 \%$ & $50 \%$ \\
\hline TAVI & $30 \%$ & $35 \%$ & $40 \%$ & $45 \%$ & $50 \%$ \\
\hline & Ano 1 & Ano 2 & Ano 3 & Ano 4 & Ano 5 \\
\hline & $50 \%$ & $40 \%$ & $30 \%$ & $20 \%$ & $10 \%$ \\
\hline Padrão terapêutico atual & $50 \%$ & $60 \%$ & $70 \%$ & $80 \%$ & $90 \%$ \\
\hline TAVI & $50 \%$ & Cenário alternativo \\
\hline
\end{tabular}

Figura 4. Taxa de penetração do implante por cateter de bioprótese valvular aórtica (TAVI) em relação ao número de pacientes elegíveis ao procedimento. 
longo do tempo horizonte de 5 anos, seria de, aproximadamente, R\$56,9 milhões. Ambos os cenários hipotéticos (cenário base e cenário alternativo) demonstraram aumento no impacto orçamentário. No cenário base, no qual os valores de distribuição da TAVI foram obtidos a partir do painel de especialistas, o impacto orçamentário é da ordem de R\$ 127 milhões em 5 anos (Figura 5).

Na Figura 6, estão representados os impactos orçamentários incrementais em 5 anos no Sistema de Saúde Suplementar em cada cenário hipotético (incorporação do TAVI) em comparação ao cenário atual (sem incorporação da TAVI).

Em ambos os cenários hipotéticos, o impacto orçamentário se deve, principalmente, ao custo da prótese valvular para implante transcateter, sendo responsável por aproximadamente $43 \%$ dos custos totais no cenário base, quando comparado a outros custos analisados no modelo (hospitalizações e gastos medicamentosos, entre outros). Já no cenário alternativo, a prótese valvular é responsável por aproximadamente 55\% dos custos, uma vez que sua distribuição de mercado é maior nesse cenário.

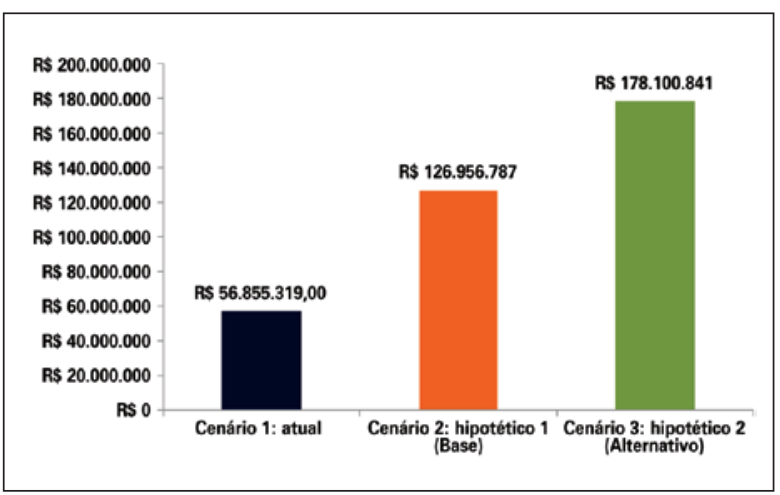

Figura 5. Resultados de impacto no orçamento acumulados no horizonte de 5 anos para os 3 cenários analisados.

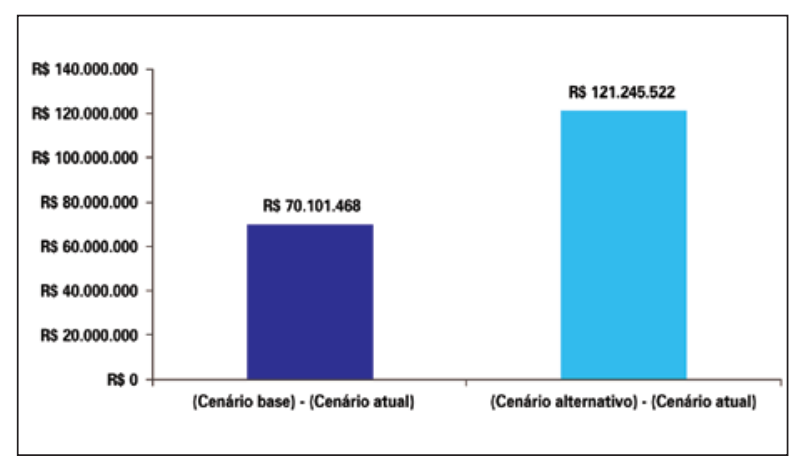

Figura 6. Impacto após a incorporação do implante por cateter de bioprótese valvular aórtica (TAVI) no Sistema de Saúde Suplementar com o cenário hipotético 1 (maior utilização do TAVI) e cenário hipotético 2 (menor utilização do TAVI).

\section{DISCUSSÃO}

Desde 1998, com a regulamentação da saúde suplementar no Brasil e a criação da Agência Nacional de Saúde Suplementar (ANS), houve crescimento sustentado nesse setor, envolvendo as operadores de planos de saúde, os beneficiários e os prestadores de serviços. Atualmente, mais de 48 milhões de brasileiros utilizam o Sistema de Saúde Suplementar. Segundo dados do Caderno de Informações da ANS, foram gastos mais de R\$ 68 bilhões pelas operadoras de planos de saúde em 2012. Em virtude de determinação legal, a ANS tem a incumbência de atualizar, a cada 2 anos, o Rol de Procedimentos e Eventos em Saúde, referência de cobertura mínima obrigatória. Nesse contexto, insere-se a necessidade de avaliar a incorporação de novas tecnologias médicas, sendo a análise econômica de grande importância para a inclusão de novos procedimentos no rol de coberturas obrigatórias.

Para Norman Daniels, ${ }^{20}$ as decisões sobre alocação de recursos financeiros no âmbito da saúde consistem em determinar: que tipo de serviços de saúde existirão numa sociedade; quem os receberá e com base em quê; quem prestará esses serviços; como será distribuída a incumbência de financiá-los; como serão distribuídos o poder e o controle desses serviços. No Brasil, essa decisão está a cargo do Ministério da Saúde. No que tange à incorporação de novas tecnologias, desde 2012, ela compete à Comissão Nacional de Incorporação de Tecnologias (CONITEC), cujas deliberações são tomadas com base na relevância e no impacto da implementação da tecnologia no sistema de saúde, bem como na existência de evidências científicas de eficácia, acurácia, efetividade, segurança e de estudos de viabilidade econômica da tecnologia proposta, em comparação às demais incorporadas anteriormente.

No âmbito da saúde suplementar, mesmo antes da criação da CONITEC, a ANS já detinha, por determinação legal, a competência de atualizar o rol de coberturas obrigatórias de procedimentos médicos, portanto, de determinar a incorporação de tecnologias médicas na ambiência a ela adstrita.

Merece ênfase, igualmente, que, em face do envelhecimento da população, a terapia em questão tem potencial para beneficiar parcela expressiva da sociedade brasileira. Segundo dados da ANS, cerca de 2,5 milhões de beneficiários têm mais de 70 anos. Com base na estimativa de prevalência de $5 \%$ dessa população apresentando EA grave, certamente fração considerável desse montante poderá ser considerada sem chance de submeter-se à cirurgia para correção da doença e, desse modo, será candidata ao TAVI. No Brasil, frise-se que o estatuto do idoso determina que essa população seja priorizada quanto ao acesso a serviços públicos e privados, aí se incluindo o direito de acesso à saúde.

Ainda em alusão aos enfermos portadores de EA grave, sem perspectivas de tratamento cirúrgico, ce- 
nário avaliado neste estudo, o TAVI representa a única alternativa terapêutica capaz de alterar o curso natural dessa grave moléstia cardiovascular, em virtude da redução significativa de mortalidade em cotejo à estratégia conservadora, conforme demonstra claramente o estudo PARTNER. ${ }^{17}$ Assim, essa terapia já foi avaliada e incorporada em diversos países. No Brasil, embora a técnica tenha sido disponibilizada desde 2008, com o registro pela Agência Nacional de Vigilância Sanitária (ANVISA) da primeira prótese valvular para implante transcateter, ainda não há previsão de cobertura obrigatória no sistema de saúde nacional, escoimando-se parcela expressiva desses enfermos idosos do tratamento cientificamente adequado.

Conforme demonstrado neste estudo, a RCEI do TAVI é entre R\$53.975,00 e R\$ 90.161,29/ano de vida salvo, variando sobretudo em virtude do preço da prótese empregada nesse procedimento. Esse número, porém, fica abaixo do limite usualmente aceito como critério para incorporação, que é de US\$ 50 mil. ${ }^{21} \mathrm{O}$ impacto orçamentário do procedimento é considerável, devido principalmente ao preço da prótese valvular. No entanto, desde 2011, quando da atualização do Rol de Procedimentos da ANS de 2010, editado por intermédio da Resolução Normativa 256, constata-se que ocorreu redução expressiva do preço dessa prótese de cerca de $40 \%$ em relação ao valor referencial utilizado neste estudo, o que deve diminuir substancialmente o impacto esperado. Em aditamento, destaque-se que, atualmente, há três próteses similares com registro na ANVISA, sendo uma delas manufaturada no Brasil; tal fato poderá tornar ainda mais favorável a RCEI do TAVI.

As limitações principais do modelo econômico desenvolvido são as usuais de estudos semelhantes feitos no país, quais sejam a inexistência de dados epidemiológicos locais, a dificuldade de coletar os custos, bem como de realizar uma projeção do uso do procedimento com base em painel de especialistas. Entretanto, essas limitações são inerentes a quaisquer estudos econômicos realizados no país e, considerando que a função deles é oferecer uma base de decisão que permita ao gestor de hoje preparar-se para o financiamento do procedimento no futuro, este estudo oferece informações importantes que devem ser consideradas no processo de adoção da tecnologia em pauta.

As diretrizes brasileiras publicadas pelo Ministério da Saúde $^{21}$ com recomendações para Avaliação de Tecnologia em Saúde não definem um limiar de RCEI elegível para incorporação, ao contrário de países europeus, do Canadá e da Austrália. No entanto, em 21 de maio de 2012, o Secretário de Atenção à Saúde do Ministério da Saúde publicou Portaria no Diário Oficial da União (Portaria SAS nº 458) com referência ao valor de US\$ 50 mil como limite superior da RCEI. Utilizando esse valor como referência, o procedimento TAVI seria custo-efetivo para o cenário brasileiro, considerando-se o limite de R\$ 100 mil, ao câmbio aproximado de US\$1,00 a R\$2,00. À guisa ainda do valor do RCEI encontrado para o TAVI neste estudo, é de bom alvitre destacar que outras tecnologias já foram incorporadas pelo Sistema de Saúde Suplementar com incremento de custo semelhante, a exemplo recente dos cardiodesfibriladores implantáveis.

Por fim, salienta-se que, em virtude da ausência de cobertura obrigatória para TAVI, tem-se propiciado uma crescente judicialização no contexto, visando à implementação do direito a essa terapia com os efeitos culminados. Dessa forma, mesmo que pela via judicial, as operadoras de planos de saúde estão sendo forçadas a suportar esses custos adicionais, e em um cenário bem mais desfavorável do ponto de vista financeiro. Assim, o valor da RCEI apurado neste estudo acaba por superestimar o real impacto orçamentário dessa incorporação no âmbito da saúde suplementar, uma vez que, efetivamente, já estão sendo alocados recursos para tal finalidade.

\section{CONCLUSÕES}

A estenose aórtica grave é enfermidade que demanda relevante gasto ao Sistema de Saúde Suplementar Brasileiro. A única terapia prevista no âmbito da Saúde Suplementar, a valvuloplastia aórtica com cateter balão, em nada altera a história natural da doença. Em contraposição, os pacientes tratados com implante por cateter de bioprótese valvular aórtica se beneficiam com a maior expectativa de vida em comparação aos pacientes que realizam apenas o tratamento conservador. Além disso, quase que a totalidade dos pacientes que utilizam o implante por cateter de bioprótese valvular aórtica, comparativamente ao tratamento padrão, demonstra maior efetividade e custo incremental, evidenciando-se robustez no resultado da análise econômica realizada.

Adicionalmente, a análise de impacto orçamentário demonstrou que a incorporação do implante por cateter de bioprótese valvular aórtica no Rol de Procedimentos e Eventos em Saúde da Agência Nacional de Saúde Suplementar, na forma como avaliado neste estudo, acarretaria estimativa de um impacto orçamentário incremental nos próximos 5 anos entre R\$ 70 milhões e R\$ 121 milhões, compatível com o de outras tecnologias já incorporadas no âmbito da saúde suplementar.

\section{CONFLITO DE INTERESSES}

Marcelo C. Queiroga recebeu auxílio institucional e é palestrante da Medtronic. Fábio Sândoli Brito Júnior é palestrante e proctor da Edwards LifeSciences e Medtronic. Rogério Sarmento-Leite é palestrante e proctor da Medtronic. Os demais autores declaram não haver conflito de interesses relacionado a este manuscrito. 


\section{REFERÊNCIAS}

1. Nkomo VT, Gardin JM, Skelton TN, Gottdiener JS, Scott CG, Enriquez-Sarano M. Burden of valvular heart diseases: a population-based study. Lancet. 2006;368(9540):1005-11.

2. Figulla L, Neumann A, Figulla HR, Kahlert P, Erbel R, Neumann T. Transcatheter aortic valve implantation: evidence on safety and efficacy compared with medical therapy. A systematic review of current literature. Clin Res Cardiol. 2011; 100(4):265-76.

3. Holmes DR Jr, Mack MJ, Kaul S, Agnihotri A, Alexander KP, Bailey SR, et al. 2012 ACCF/AATS/SCAI/STS expert consensus document on transcatheter aortic valve replacement: developed in collaboration with the American Heart Association, American Society of Echocardiography, European Association for Cardio-Thoracic Surgery, Heart Failure Society of America, Mended Hearts, Society of Cardiovascular Anesthesiologists, Society of Cardiovascular Computed Tomography, and Society for Cardiovascular Magnetic Resonance. Ann Thorac Surg. 2012; 93(4):1340-95.

4. Brady ST, Davis CA, Kussmaul WG, Laskey WK, Hirshfeld JW Jr, Herrmann HC. Percutaneous aortic balloon valvuloplasty in octogenarians: morbidity and mortality. Ann Intern Med. 1989;110(10):761-6

5. Bonow RO, Carabello BA, Kanu C, de Leon AC Jr, Faxon DP, Freed MD, et al. ACC/AHA 2006 guidelines for the management of patients with valvular heart disease: a report of the American College of Cardiology/American Heart Association Task Force on Practice Guidelines (writing committee to revise the 1998 Guidelines for the Management of Patients With Valvular Heart Disease): developed in collaboration with the Society of Cardiovascular Anesthesiologists: endorsed by the Society for Cardiovascular Angiography and Interventions and the Society of Thoracic Surgeons. Circulation. 2006;114(5):e84-231.

6. Brito Junior FS, Lemos PA, Siqueira D, Sarmento-Leite R, Mangione JA, Carvalho LA, et al. Consenso de Especialistas sobre o implante por cateter de biopróteses valvares para o tratamento da estenose aórtica de alto risco cirúrgico: Relato da Sociedade Brasileira de Hemodinâmica e Cardiologia Intervencionista. Rev Bras Cardiol Invasiva. 2011;19(2):200-7.

7. Vahanian A, Baumgartner H, Bax J, Butchart E, Dion R, Filippatos G, et al. Guidelines on the management of valvular heart disease: the Task Force on the Management of Valvular Heart Disease of the European Society of Cardiology. Eur Heart J. 2007;28(2):230-68.

8. Carabello BA. Evaluation and management of patients with aortic stenosis. Circulation. 2002;105(15):1746-50.

9. Iung B, Cachier A, Baron G, Messika-Zeitoun D, Delahaye $\mathrm{F}$, Tornos $\mathrm{P}$, et al. Decision-making in elderly patients with severe aortic stenosis: why are so many denied surgery? Eur Heart J. 2005;26(24):2714-20.
10. Ben-Dor I, Pichard AD, Gonzalez MA, Weissman G, Li Y, Goldstein SA, et al. Correlates and causes of death in patients with severe symptomatic aortic stenosis who are not eligible to participate in a clinical trial of transcatheter aortic valve implantation. Circulation. 2010;122(11 Suppl):S37-42.

11. Tarasoutchi F, Montera MW, Grinberg M, Barbosa MR, Piñeiro DJ, Sánchez CRM, et al. Diretriz Brasileira de Valvopatias SBC 2011 / I Diretriz Interamericana de Valvopatias - SIAC 2011. Arq Bras Cardiol. 2011;97(5 Supp 1):1-67.

12. Sehatzadeh S, Doble B, Xie F, Blackhouse G, Campbell K, Kaulback K, et al. Transcatheter aortic valve implantation (TAVI) for treatment of aortic valve stenosis: an evidence-based analysis (part B). Ont Health Technol Assess Ser. 2012; 12(14):1-62.

13. Cribier A, Eltchaninoff $\mathrm{H}$, Bash A, Borenstein N, Tron C, Bauer $F$, et al. Percutaneous transcatheter implantation of an aortic valve prosthesis for calcific aortic stenosis: first human case description. Circulation. 2002;106(24):3006-8.

14. Gilard M, Eltchaninoff H, lung B, Donzeau-Gouge P, Chevreul K, Fajadet J, et al. Registry of transcatheter aortic-valve implantation in high-risk patients. N Engl J Med. 2012;366(18):1705-15.

15. National Institute for Health and Care Excellence. IPG421 Transcatheter aortic valve implantation for aortic stenosis: guidance [Internet]. 2012 [cited 2013 June 22]. Available from: http://guidance.nice.org.uk/IPG421/Guidance/pdf/English

16. National Health Committee. Briefing report: Transcatheter Aortic Valve Implantation (TAVI). [Internet]. Wellington: NHC; 2012 [cited 2013 June 22]. Available from: http://nhc.health. govt.nz/system/files/documents/publications/tavi-briefing-reportsept2012.pdf

17. Leon MB, Smith CR, Mack M, Miller DC, Moses JW, Svensson LG, et al.; PARTNER Trial Investigators. Transcatheter aorticvalve implantation for aortic stenosis in patients who cannot undergo surgery. N Engl J Med. 2010;363(17):1597-607.

18. Reynolds MR, Magnuson EA, Wang K, Lei Y, Vilain K, Walczak $J$, et al. Cost-effectiveness of transcatheter aortic valve replacement compared with standard care among inoperable patients with severe aortic stenosis: results from the placement of aortic transcatheter valves (PARTNER) trial (Cohort B). Circulation. 2012;125(9):1102-9.

19. O'Neill WW. Predictors of long-term survival after percutaneous aortic valvuloplasty: report of the Mansfield Scientific Balloon Aortic Valvuloplasty Registry. J Am Coll Cardiol. 1991; 17(1):193-8

20. Daniels N. Just health care Just health care. New York: Cambridge University Press; 1985.

21. Brasil. Ministério da Saúde. Rede Brasileira de Avaliação em Tecnologias de Saúde. Métodos e diretrizes [Internet]. Brasília; 2013 [citado 2013 jun. 22]. Disponível em: http://200.214.130.94/ rerats/diretriz.php 
$\mathbf{N}$ a edição de setembro de 2013 da Revista Brasileira de Cardiologia Invasiva (RBCl 21-3), no artigo "Implante por Cateter de Bioprótese Valvular Aórtica para Tratamento de Estenose Valvar Aórtica Grave em Pacientes Inoperáveis sob Perspectiva da Saúde Suplementar - Análise de Custo-Efetividade", de autoria de Queiroga et al. (Rev Bras Cardiol Invasiva. 2013;21(3):438-9), onde se lê:

\section{Na página 213}

Para o cenário padrão, no qual o custo da TAVI foi estipulado em R\$ 65 mil, o valor da RCEI (custo/ano de vida salvo) em 5 anos foi de $\mathrm{R} \$ 72.520,65$. Alterando-se o tempo horizonte para 10 anos, esse valor diminuiu para $\mathrm{R} \$ 41.653,01$.

Leia-se: Para o cenário padrão, no qual o custo da prótese valvular foi estipulado em R\$ 65 mil, o valor da RCEI (custo/ano de vida salvo) em 5 anos foi de $\mathbf{R} \mathbf{9 0 . 1 6 1 , 2 9 . ~ A l t e r a n d o - s e ~ o ~ t e m p o ~ h o r i z o n t e ~ p a r a ~} 10$ anos, esse valor diminuiu para $\mathbf{R} \mathbf{5 5 . 1 3 0 , 8 4}$.

\section{Nas páginas 213-214}

In a standard scenario, where the cost of TAVI was estimated as R\$ 65 millions, the ICER value (cost/year of life saved) in 5 years was $R \$ 72,520.65$. When the time horizon was adjusted for 10 years, this amount decreased to $R \$ 41,653.01$.

Leia-se: In a standard scenario, where the cost of the valvular prosthesis was estimated as $R \$ \mathbf{6 5 . 0 0 0 , 0 0}$, the ICER value (cost/year of life saved) in 5 years was $\mathbf{R} \mathbf{9 0 . 1 6 1 , 2 9}$. When the time horizon was adjusted for 10 years, this amount decreased to $\mathbf{R} \mathbf{\$} \mathbf{5 5 . 1 3 0 , 8 4}$.

\section{Na página 217}

Adicionalmente, alterou-se o valor da prótese valvular entre R\$30 mil e R\$ 65 mil (valor do caso base). Os resultados de RCEI dessa análise no tempo horizonte de 5 anos, com essa alteração do custo da prótese valvular, variaram entre $R \$ 49.770,00$ e $R \$ 85.957,00$. Os valores para cada alteração estão descritos na Figura 3.

Leia-se: Adicionalmente, alterou-se o valor da prótese valvular entre R\$30 mil e R\$ 65 mil (valor do caso base). Os resultados de RCEI dessa análise no tempo horizonte de 5 anos, com essa alteração do custo da prótese valvular, variaram entre $\mathbf{R} \mathbf{\$} \mathbf{5 3 . 9 7 5 , 0 0}$ e $\mathbf{R} \mathbf{9 0 . 1 6 1 , 2 9}$. Os valores para cada alteração estão descritos na Figura 3.

\section{Na página 219}

Conforme demonstrado neste estudo, a RCEI do TAVI é entre R\$ 49.770 e R\$ 85.957/ano de vida salvo, variando sobretudo em virtude do preço da prótese empregada nesse procedimento. Esse número, porém, fica abaixo do limite usualmente aceito como critério para incorporação, que é de US\$50 mil.

Leia-se: Conforme demonstrado neste estudo, a RCEI do TAVI é entre $\mathbf{R} \mathbf{5 3 . 9 7 5 , 0 0}$ e $\mathbf{R} \$ \mathbf{9 0 . 1 6 1 , 2 9}$ /ano de vida salvo, variando sobre tudo em virtude do preço da prótese empregada nesse procedimento. Esse número, porém, fica abaixo do limite usualmente aceito como critério para incorporação, que é de US\$ 50 mil. 